\title{
Telemetric ECG Monitoring During Physical Activity in Field Tests
}

Boris Siraiy, Roman Trobec

Institut "JoZef Stefan"

Jamova cesta 39

1000 Ljubljana, Slovakia

boris.siraiy@hotmail.com,roman.torbec@ijs.si

ABSTRACT: In this paper we show how it is possible to measure ECG signal with telemetric ECG body-sensor during physical activity in regular conditions. Based on previous studies we choose position and type of fixation for sensor. Participant made three different tests, first test was shuttle run test where speed of running was increased every minute, second test was Cooper $2400 \mathrm{~m}$ test, and third test was $100 \mathrm{~m}$ maximal sprint. We measure ECG signal in all three tests and analyse it with special software for Holter analysis.

Keywords: ECG Body Sensors, Shuttle Run, Cooper 2400 m, 100 m Sprint, Healthcare

DOI:10.6025/ed/2019/8/1/1-6

Received: 17 August 2018, Revised 5 October 2018, Accepted 19 October 2018

(C) 2019 DLINE. All Rights Reserved

\section{Introduction}

Nowadays sudden cardiac death presents extremely high risk for sudden death of professional sportsmen and recreational athletes. Cardiovascular disease causes more than $90 \%$ of sudden deaths during physical activity [1]. Two France studies reported a daily incidence of 3 sudden deaths and 4 myocardial infarctions during physical activity in the general population $[2,3]$. Studies from USA shows sudden cardiac death incidence between sportsmen in high schools and colleagues is 1:200.000 per year $[4,5,6]$, while European studies shows that incidence is even higher, 1- 1,6:100.000 [7,8]. Considering the fact that 55-80\% of the athletes who died of sudden cardiac death had no prior symptoms of heart disease [9], the question is what else should be done for the prevention of sudden cardiac death, and to detect the people who has increased risk of sudden cardiac death.

Telemetric ECG body sensor used in this paper was already used in previous pilot studies made during light physical activity [10], and in maximal laboratory exercise stress test (EST) [11]. Since an EST with ECG monitoring is usually performed in laboratory conditions, our goal is to determine whether it is possible to measure an ECG during regular sport activities, because it will offer a significant advantage for the prevention of cardiovascular accidents [12]. 
Aim of this study was to evaluate body-sensor ECG signals during three different field tests, and to analyse the quality of the recorded ECG signal. Next aim was to determine if different types of sensor fixation influenced the ECG signal. The use of a telemetric ECG during physical activity could be very beneficial for medical doctors and sports scientists, and the most of all for professional and recreational athletes.

\section{Material and Methods}

\subsection{Study Population}

In this study was included one participant, age 24 years. He was a student at the Faculty of Sport and Physical Education at the University of Belgrade. He was healthy and without known previous cardiac problems. Before the tests the study purpose, and its protocol were explained to him, and he signed consent for participation in the study.

\subsection{Experimental Setup}

ECG measurements during the field tests were made with wireless ECG body sensors Savvy (Saving d.o.o., Ljubljana, Slovenia) [13], which is a certified medical device, described in detail in the study of Trobec et al. [14]. The body sensor is light and nonobstructive for users, which allows long-term exercise ECG measurements. The sensor is fixed on the body via two selfadhesive skin electrodes. An Android application, MobECG, which runs on a smartphone, captures and displays the measured data and saves it in the smart phone's memory for further processing.
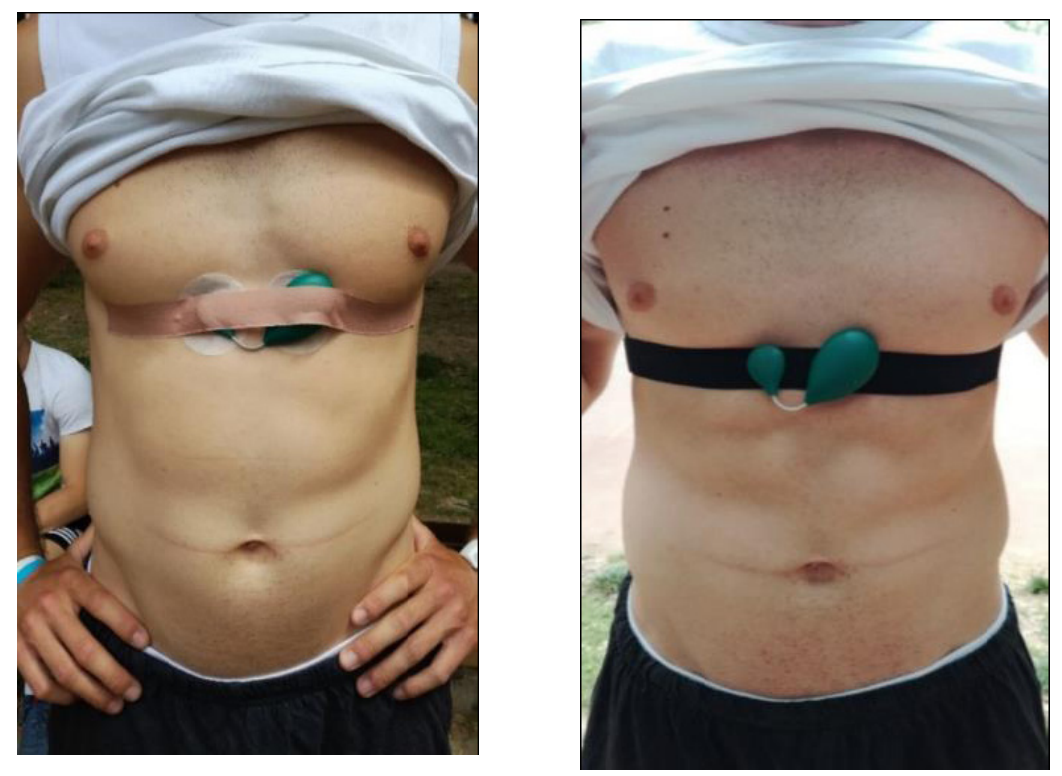

Figure 1. Sensor position and type of sensor fixation in the first test (left-hand) and in the second test (right-hand)

The sensor position should be close to the heart to obtain appropriate amplitude of ECG signal. In addition, its position should avoid large muscles, due to the signals from the electrical muscular activity (EMG) that could disturb the ECG [14,15]. In this study was used position Left Inferior (LI), shown as most adequate in previous study [11]. In the LI position the sensor electrodes are at the positions V1 and V2 of standard precordial leads, and the sensor is translated by approximately $10 \mathrm{~cm}$, below the xiphoid, where the influence of muscular disturbances is expected to be minimal (see Figure 1). The ECG electrodes were positioned $5 \mathrm{~cm}$ apart [16]. Before the positioning, we cleaned the skin of the subjects with diluted ethanol.

The participant made six tests, two shuttle run, two Cooper $2400 \mathrm{~m}$, and two $100 \mathrm{~m}$ sprint test. In the first test the ECG body sensor were fixed with self-adhesive medical Omniplast 2,5 cm tape (Paul Hartmann AG, Heidenheim, Germany), specially designed to fix Holter electrodes. Both parts of the sensors were fixed together with one, approximately 40-cm-long strip of tape. In the second test sensor were fixed with standard Polar belt made for measurements of the heart rate (HR) with Polar system.

The measured ECG data were continuously stored in the mobile phone memory and transferred to the personal computer. The ECG 
analysis were made with medically certified Holter interpretation software QuickReader ${ }^{\circledR}$ AFT-1000 (Holter Supplies, Paris, France).

\subsection{Measurement Protocol}

Every test was made in a separate day, except $100 \mathrm{~m}$ sprints where both tests were made in the same day, with 30 min pause in between tests. Before performing the tests, the electrodes and sensors were positioned at the LI position and fastened with selfadhesive tape. After that the participant sat down and waited for 5 minutes, while the ECG was recorded. After 5 minutes the participant started with the test. When the test was finished the participant sat down and rested for 5 minutes, while the ECG was still recording. The next day the participant took part in a second test. In the second test sensors were fixed with Polar belt. The ECG recording protocol was the same as on the previous day. The next type of test, under the same protocol, were performed in the next 2 days. The study was conducted in accordance with the ethical standards of the Faculty of Sport and Physical Education (IRB approval No. 02-1359/18-2), University of Belgrade, and the Helsinki Declaration.

\section{Results}

In the first shuttle run test participant successfully made 11 minutes of the test (11/2) with the maximal heart rate (HR) 196 beats per minute (bpm), while in second test he successfully made 10 minutes of the test (10/10) with the maximal HR $192 \mathrm{bpm}$. In the first Cooper test he run 11:49 minute, with the maximal HR $192 \mathrm{bpm}$, and in the second test he run 13:10 min, with the maximal HR 193 $\mathrm{bpm}$. In the $100 \mathrm{~m}$ sprint test he run 13:45 sec in the first test, with maximal HR $172 \mathrm{bpm}$, while in second test he run 13:84 sec, with the maximal HR $179 \mathrm{bpm}$.

Analyse of the signal show that ECG signal was of adequate quality in both shuttle run tests, while in Cooper and in $100 \mathrm{~m}$ sprint test signal was of adequate quality only in second test when sensor was fixed with a Polar belt. An example of screenshot of a typical HR signal in bpm, and correct QRS complex detection of a signal which is of adequate quality for interpretation and for further analysis is shown in Figure 2.

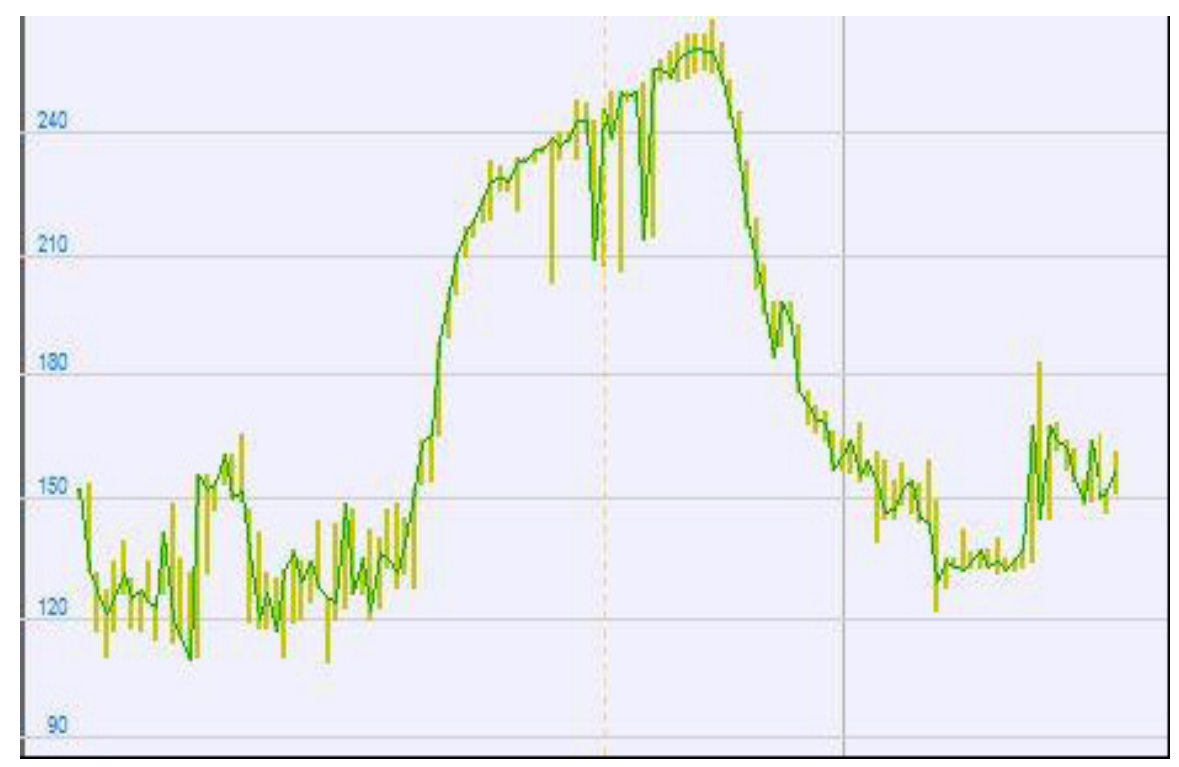

Figure 2. An example of adequate quality signal

An example of screenshot of a signal which is not of adequate quality for interpretation and for further analysis is shown in Figure 3.

The computerized heart-rate analysis was successful for most of the time during both shuttle run tests and during Cooper and 100 $\mathrm{m}$ tests when sensor was fixed with Polar belt. During the visual inspection we also found erroneously detected QRS complexes, using the Holter interpretation software, often because of the excessive artefacts that come from the intense activity (right-hand part of Figure 4). 


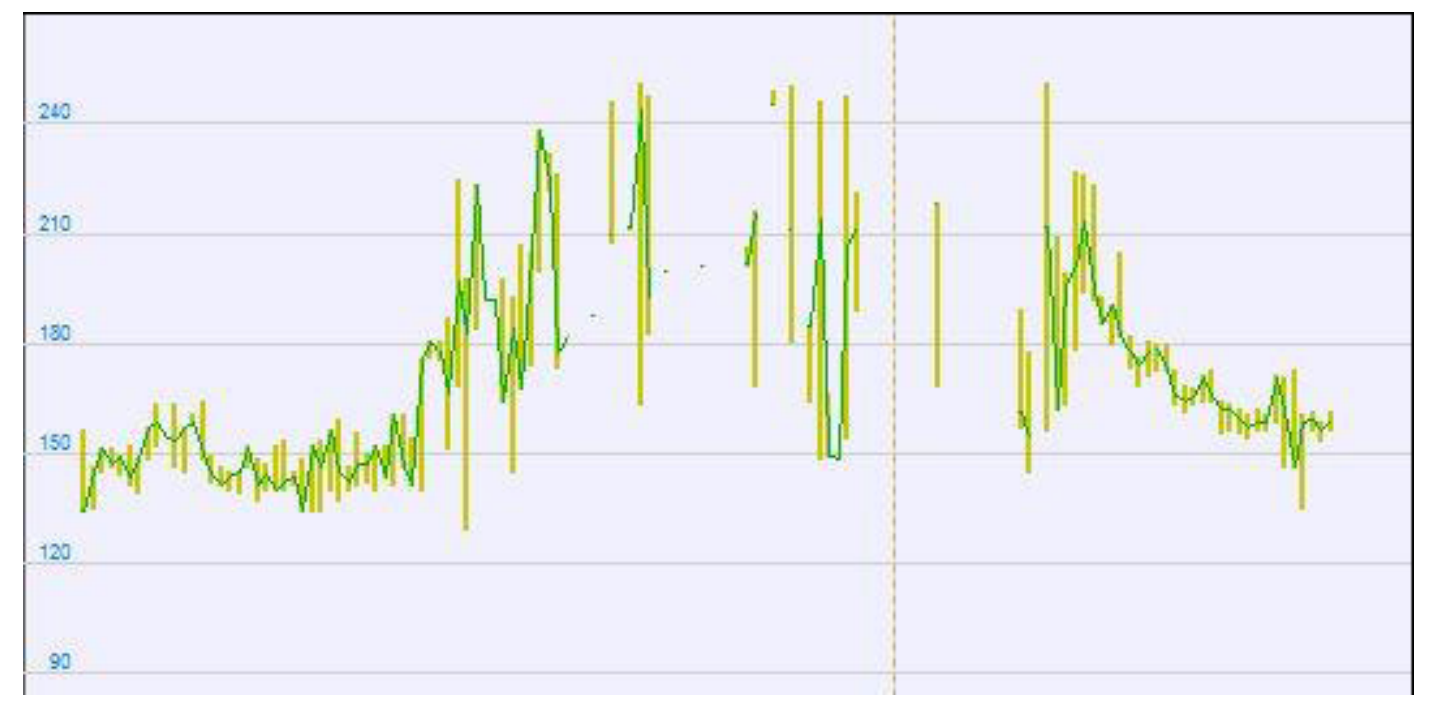

Figure 3. An example of a signal which cannot be interpreted
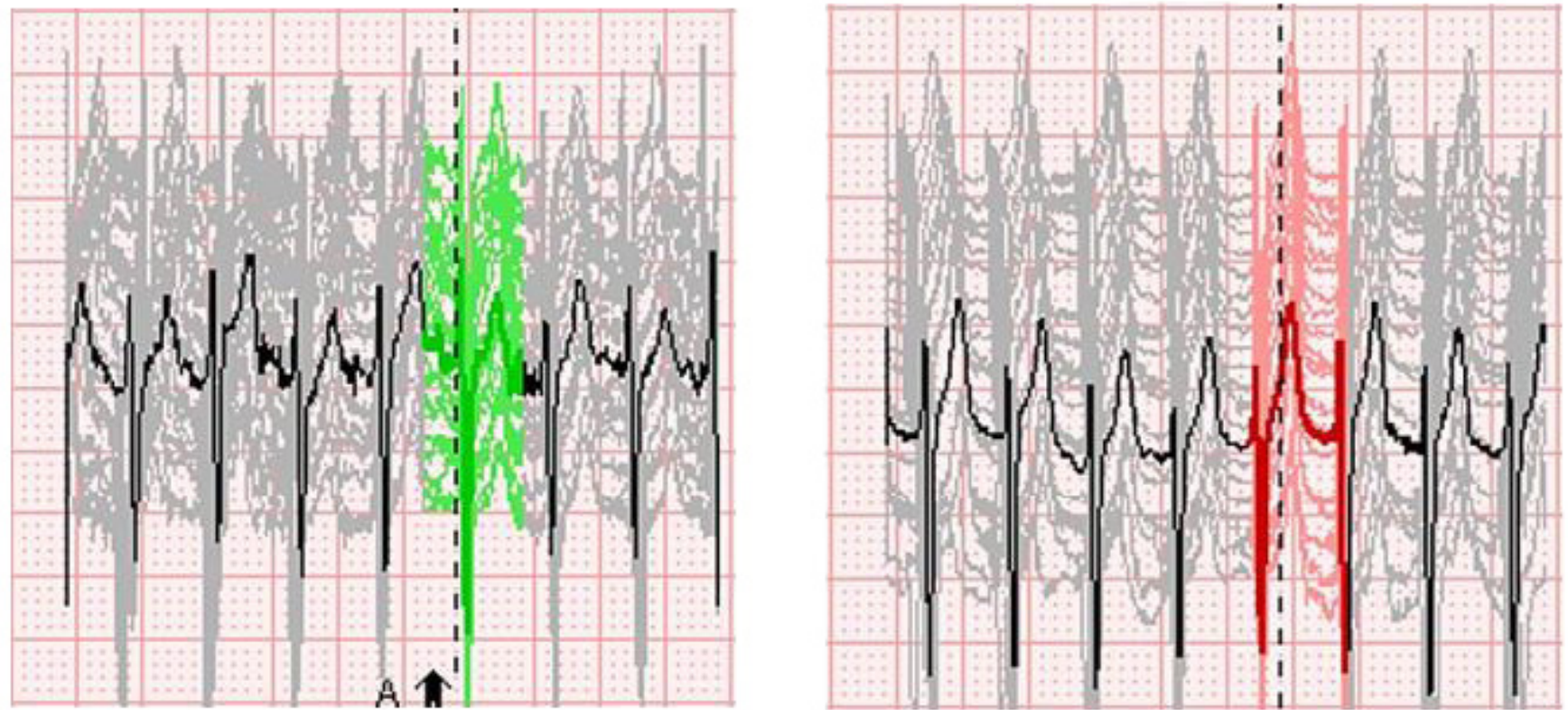

Figure 4. Visualization of the detected heart beats using the Holter interpretation software QR. The correctly interpreted QRS complexes are shown in the left-hand panel. An example of the erroneously interpreted QRS complexes is shown in the righthand panel

\section{Discussion}

This paper show that a wireless ECG body sensor can be used for non-obstructive measurements of an ECG during some regular physical activities. The aim of this study was to check if wireless ECG body sensors can be used during field tests. The results show that the type of fixation and the type of test influence the results. When sensor was fixed with Polar belt signal was of adequate quality during entire test, but when sensor was fixed with Omniplast tape the signal was of adequate quality only in shuttle run test, when the speed of running starts with $8.5 \mathrm{~km} / \mathrm{h}$ and increases $0.5 \mathrm{~km} / \mathrm{h}$ every minute, while in tests when speed of running was constantly high the signal was not acceptable. In that case maximal speed of running in shuttle run test was 
between 13.5 and $14.0 \mathrm{~km} / \mathrm{h}$, and this was very similar to previous laboratory study which confirm that ECG signal on a treadmill was of adequate quality for speeds of running up to $13.5 \mathrm{~km} / \mathrm{h}$.

The diagnostic ability of the ECG body sensor, used in this study, has been compared in various previous pilot studies with other similar devices [14] and with standard 12-lead ECG [14,17]. Previous studies shown that ECG body sensor, even so simple, can also detect most of the arrhythmic events, e.g., atrial or ventricular fibrillation, exstrasystole, tachycardias, bradycardias, etc. The analysed measurement methodology can provide basic information about the heart rhythm's status. In the case of any detected abnormality the users can be directed to further diagnostics with a standard stress test and medical personnel.

\section{Conclusion}

The presented results are a motivation for further study, where more participants will be included. With such a telemetric approach it might be possible, to the best of our knowledge for the first time, to routinely measure ECG signals in real conditions, e.g., when users are running in nature, during a significant activity. In the presented paper an appropriate method of sensor fixation was found; however, to confirm this conclusion study should be extended, and heterogenous group of participants, e.g., male and female, should be included.

\section{Acknowledgments}

This paper was supported by Slovenian Research Agency under the Grant P2-0095.

\section{References}

[1] Mazi, S., Ili, V., eli, M., Arandjelovi, A. (2011). Sudden cardiac death in young athletes. Srp. Arh. Celok. Lek., 139, 394-401.

[2] Chevalier, L., Hajjar, M., Douard, H., Charief, A., Dindard, J.M., et al. (2009). Sports-related acute cardiovascular events in a general population. A French prospective study. Eur. J. Cardiovasc. Prev. Rehabil., 16, 365-370.

[3] Marijon, E., Uy-Evanado, A., Reinier, K., Teodorescu, C., Narayanan, K., Jouven, X., Gunson, K., Jui, J., Chugh, S.S. (2015). Sudden cardiac arrest during sports activity in middle age. Circulation, 131, 1384-1391.

[4] Maron, B.J., Gardin, J.M., Flack, J.M., Gidding, S.S., Kurosaki, T.T., Bild, D.E. (1995). Prevalence of hypertrophic cardiomyopathy in a general population of young adults: echocardiographic analysis of 4111 subjects in the CARDIA study. Circulation, 92, 785-789.

[5] Maron, B.J., Shirani, J., Poliac, L.C., Mathenge, R., Roberts, W.C., Mueller, F.O. (1996). Sudden death in young competitive athletes: clinical, demographic, and pathological profiles. JAMA, 276, 199-204.

[6] Pedoe, D.T. (2000). Sudden cardiac death in sport-spectre or preventable risk? Br. J. Sports Med., 34, 137-140.

[7] Epstein, S.E., Maron, B.J. (1986). Sudden death and the competitive athlete: perspectives on preparticipation screening studies. Journal of the American College of Cardiology, 7, 220-230.

[8] Pigozzi, F., Spataro, A., Fagnani, F., Maffulli, N. (2003). Preparticipation screening for the detection of cardiovascular abnormalities that may cause sudden death in competitive athletes. British Journal of Sports Medicine, 37, 4-5.

[9] Drezner, J.A., Courson, R.W., Roberts, W.O., Mosesso, V.N., Link, M.S., Maron, B.J. (2007). Inter-association task force recommendations on emergency preparedness and management of sudden cardiac arrest in high school and college athletic programs: a consensus statement, J. Athl. Train., 42, 143-158.

[10] 'iraiy, B., Stani , U.J., Poplas-Suši , A., Katki, Z. (2018). Impact assessment of the mornint gymnastics “1000 movements" via ECG and sport tests. $41^{\text {st }}$ Int Conven. on Inform. and Communic. Technol., Electron. and Microelectron. (MIPRO), 284-288.

[11] 'iraiy, B., Ili, V., Trobec, R. (2018). Evaluating telemetric ECG body sensor signal in exercise stress test: Pilot study. 41st Int. Conven. on Inform. and Communic. Technol., Electron. and Microelectron., 289-294.

[12] Casa, D.J., Guskiewicz, K.M., Anderson, S.A., Courson, R.W., Heck, J.F., et al. (2012). National Athletic Trainers' Association Position Statement. Preventing Sudden Death in Sports. J. Athl. Train., 47 (1), 96-118.

[13] www.savvy.si, last time visited on 15. September, 2018. 
[14] Trobec, R., Tomaši, I., Rashkovska, A., Depolli M., Avbelj, V. (2018). Body sensors and electrocardiography, SpringerBriefs in Applied Sciences and Technology.

[15] Clancy, E.A., Morin, E.L., Merletti, R. (2002). Sampling, noise-reduction and amplitude estimation issues in surface electromyography. J. Electromyogr. Kinesiol., 12 (1) 1-16.

[16] Kania, M., Rix, H., Fereniec, M., Fernandez, H.Z., Janusek, D., Mroczka, T., Stix G., Maniewski, R. (2014). The effect of precordial lead displacement on ECG morphology. Med. Biol. Eng. Comput., 52, 109-119.

[17] Trobec, R., Avbelj, V., Rashkovska, A. (2014). Multifunctionality of Wireless Body Sensors. Transac. Int. Res., 10 (1) 23-27. 